\title{
Personalised Approaches in Treating Early-Stage Hodgkin Lymphoma
}

DOI:

10.1136/bmj.h2927

Document Version

Final published version

Link to publication record in Manchester Research Explorer

\section{Citation for published version (APA):}

Illidge, T. (2020). Personalised Approaches in Treating Early-Stage Hodgkin Lymphoma. Internal Medicine Research - Open Journal, 5(3), 1. https://doi.org/10.1136/bmj.h2927

\section{Published in:}

Internal Medicine Research - Open Journal

\section{Citing this paper}

Please note that where the full-text provided on Manchester Research Explorer is the Author Accepted Manuscript or Proof version this may differ from the final Published version. If citing, it is advised that you check and use the publisher's definitive version.

\section{General rights}

Copyright and moral rights for the publications made accessible in the Research Explorer are retained by the authors and/or other copyright owners and it is a condition of accessing publications that users recognise and abide by the legal requirements associated with these rights.

\section{Takedown policy}

If you believe that this document breaches copyright please refer to the University of Manchester's Takedown Procedures [http://man.ac.uk/04Y6Bo] or contact uml.scholarlycommunications@manchester.ac.uk providing relevant details, so we can investigate your claim.

\section{OPEN ACCESS}




\title{
Personalised Approaches in Treating Early-Stage Hodgkin Lymphoma
}

\author{
Tim Illidge* and Elizabeth H Phillips \\ Division of Cancer Sciences, Faculty of Biology, Medicine and Health, University of Manchester, NIHR Biomedical Research Centre, Manchester Academ-
} ic Health Sciences Centre, Christie Hospital NHS Foundation Trust, Manchester, UK

${ }^{*}$ Corresponding author: Tim Illidge, Division of Cancer Sciences, Faculty of Biology, Medicine and Health, University of Manchester, NIHR Biomedical Research Centre, Manchester Academic Health Sciences Centre, Christie Hospital NHS Foundation Trust, Manchester, UK; Email: tim.illidge@manchester.ac.uk

Received: June 12, 2020; Accepted: June 24, 2020; Published: July 01, 2020

\section{Editorial}

Early-stage Classical Hodgkin Lymphoma (HL) is now one of the most curable malignancies and one of the success stories of modern haemato-oncology. Great progress has been made since X-rays were used as one of the first examples of non-surgical cancer treatment in 1901 by Pusey. More than half a century later, the development of wide field megavoltage irradiation techniques to multiple lymph node chains led by Peters in the 1950's and Kaplan in the 1960's were transformational and meant for the first time there were longterm survivors treated for HL $[1,2]$. Increasing use of systemic chemotherapy in the 1970's improved outcomes yet further. However, this improvement in tumour control came at a cost and follow-up in these long-term survivors led to the concerning observations that more patients were dying in later life from the consequences of their treatment than from recurrence of their HL $[3,4]$.

In this century, the approach towards management of early-stage HL has therefore increasingly focused on improving the quality of long-term survival by aiming to decrease treatment-induced mortality and morbidity whilst at the same time maintaining long-term disease control. The aims of treatment are to balance the best chance of cure against the risks of late side effects that may lead to reduced longterm survival and impaired quality of life. Striking the right balance in this treatment goal has in turn led to the ambition to develop a personalised approach to treating HL. However there remain many unmet challenges in achieving the goal of personalised therapy for HL and some of these will be outlined in this brief commentary. These challenges include moving beyond the well-established pretreatment clinical risk stratification approaches, integrating $\left[{ }^{18} \mathrm{~F}\right]$ fluorodeoxyglucose ([ $\left.\left.{ }^{18} \mathrm{~F}\right] \mathrm{FDG}\right)$ Positron Emission Tomography (PET) response-adjusted therapy, integrating individual patient characteristics (age, sex, site of disease) alongside emerging biological biomarkers in the decision making.

\section{Risk-adapted Strategies: Pre-treatment Clinical Risk Stratification and Combined Modality Therapy}

Pre-treatment staging and clinical risk stratification have been important tools for treatment decision making in early-stage HL over recent decades. Most international groups have used clinical risk factors, such as number of involved nodal sites, Erythrocyte Sedimentation Rate (ESR), and the presence of mediastinal bulk or B-symptoms, to differentiate early-stage HL into favourable and unfavourable risk groups with different treatment approaches. A series of large randomised trials conducted by the German Hodgkin Study Group (GHSG) and European Organisation for the Research and Treatment of Cancer (EORTC) have used clinical risk stratification to optimise CMT approaches for early-stage HL [5-7]. The GHSG HD10 trial showed that 2 cycles of ABVD and 20Gy Involved Field Radiation Therapy (IFRT) delivered excellent long-term tumour control, with freedom from treatment failure of $91.1 \%$ at 5 years [8]. This CMT approach became widely established as a standard treatment approach in early-stage favourable HL. For early-stage unfavourable $\mathrm{HL}$, the results from the GSHG HD11 using 4 x ABVD and 30 Gy IFRT provided $83 \%$ long-term Progression-Free Survival (PFS), with room for further clinical improvements [9]. In an attempt to improve outcomes further, the GHSG HD14 trial investigated intensification of chemotherapy with 2 cycles of BEACOPP ${ }_{\text {escalated }}$ plus $2 \times$ ABVD $(2+2)$. This approached improved PFS in early-stage unfavourable HL, with an improved 5 year PFS difference of $6.2 \%$ over the "standard arm". However, this " $2+2$ " approach was associated with more acute haematological toxicity and no difference in long-term toxicity or overall survival, therefore this more intensified approach has not been widely adopted.

Whilst pre-treatment clinical risk stratification can be used to guide treatment selection within validated treatment protocols, there are a number of limitations. These include the fact that all adverse risk factors are given equal weight in scoring, albeit that mediastinal bulk appears to have influenced treatment decisions perhaps more than other risk factors [10]. In view of these limitations, pre-clinical risk stratification is less frequently applied in many UK and US early-stage HL clinical trial protocols and patients with mediastinal bulk and B-symptoms are excluded from early stage protocols and treated as advanced-stage disease. Secondly, the prognostic value of pre-clinical risk stratification in the current era of PET- risk adapted therapy has become less clear. By way of example the in the UK NCRI PET adapted "RAPID" trial, the PET response after 3 cycles of ABVD was more important than preclinical risk factors with no difference in outcomes seen between patients who initially presented with favourable- and 
unfavourable-risk disease [11]. Importantly patients with mediastinal bulk and B-symptoms were excluded from this trial. Finally, risk stratification does not take account of other baseline variables that may have utility in early-stage HL, including Maximum Tumour Diameter (MTD) and metabolic tumour volume [12-14].

Emerging tissue and serum biomarkers may in future help to refine pre-treatment risk stratification and develop precision medicine strategies that reflect underlying disease biology [15]. Circulating biomarkers such as serum Thymus Activation-Related Cytokine (TARC) and circulating tumour DNA have prognostic value in HL but have not yet been evaluated within early-stage HL protocols $[16,17]$; further assessment and standardisation is required before wider clinical application.

\section{Response-adapted Strategies: PET-directed Treatment}

PET response assessment has emerged as one of the most powerful prognostic tools in HL over recent decades [18]. A number of large, randomised trials have informed the use of PET-adapted treatment approaches, including the UK NCRI RAPID trial, EORTC H10, US intergroup 50604 Phase II trial and GHSG HD16 trials [19-22]. The principal focus of these clinical trials has been to investigate the utility of PET as an imaging biomarker to deliver response-adjusted therapy with Chemotherapy Alone (CA) for those who achieve Complete Metabolic Response (CMR). In this way, the aim has been to potentially avoid RT and further reduce RT -related long-term toxicity. The first trial to report was the RAPID trial, which included 602 patients with stage I/IIA HL and no mediastinal bulk [22]. Patients received 3 cycles of ABVD followed by PET response assessment. Those achieving a CMR (defined as Deauville score 1-2) were randomly assigned to receive 30 Gy IFRT or no further treatment. Approximately twothirds of patients enrolled had a favourable risk profile according to GHSG or EORTC risk classification. PET-negative patients in the intent-to-treat and per-protocol cohorts had PFS differences of 3.8\% (3-year PFS, $94.6 \%$ vs $90.8 \%$ ) and 6.3\% (3-year PFS, 97.1\% vs 90.8\%) favouring consolidative IFRT, respectively.

In a similar fashion, the EORTC/Fondazione Italiana Linfomi performed the HD10 trial in both Early stage Favourable (F) and unfavourable $(\mathrm{U})$ patients. In the standard arm, all patients received either $3(\mathrm{~F})$ or 4 cycles (U) of ABVD followed by 30Gy ISRT. In the experimental arm, patients achieving CMR after 2 cycles ABVD received chemotherapy alone, with a total of $4(\mathrm{~F})$ or $6(\mathrm{U})$ cycles of ABVD. In a pre-planned interim analysis of PET-negative patients, futility of the trial was declared by the independent data monitoring committee because of an increased number of HL-related events in the non-RT arms [23]. The 5-year PFS rates with and without RT were $99.0 \%$ and $87.1 \%$, respectively for favourable-risk disease, and $92.1 \%$ and $89.6 \%$, for unfavourable-risk disease. The GHSG HD16 trial compared 2 cycles of ABVD alone with 2 ABVD plus 20Gy IFRT in patients with favourable-risk early-stage HL that achieved CMR after chemotherapy [21]. Again, there was a PFS difference in favour of CMT, with 5-year PFS rates of $93.4 \%$ with CMT and $86.1 \%$ with ABVD.

Although non-inferiority of chemotherapy alone compared with CMT could not be demonstrated, it is important to note that overall survival rates were excellent, uniformly exceeding 95\% for both CMT and chemotherapy alone at 5 years. In most studies, non-HL deaths were a greater risk and outnumbered HL-related deaths, irrespective of treatment approach [21,22]. Follow-up for these studies is still relatively short and it remains to be seen whether mortality from late RT-related toxicity has an impact on survival over the decades to come, therefore the optimum approach remains unclear.

To address whether intensified therapy can improve outcomes in early-stage HL, the $\mathrm{H} 10$ trial evaluated a response-adapted approach based on PET assessment after 2 cycles of ABVD. In the standard arm, PET-positive patients (Deauville score 3-5) continued with ABVD to receive a total of $4(\mathrm{~F})$ or $6(\mathrm{U})$ cycles followed by 30Gy INRT, whilst in the experimental arm, PET-positive patients were switched to an intensified treatment with 2 x BEACOPP escalated plus 30 Gy INRT. Outcomes clearly favoured the experimental arm, with 5-year PFS rates of $90.6 \%$ compared with $77.4 \%$ for PET-positive patients in the standard arm and a trend towards improved overall survival $(96.0 \%$ versus $89.3 \%$, respectively, $\mathrm{p}=0.062$ ) [19]. This approach has been adopted as standard of care in many centres, although the degree of PET-positivity is an important consideration. An analysis of PET-positive patients in the RAPID protocol has shown that patients with stage I/IIA non-bulky HL that achieve Deauville score 3 or 4 have excellent outcomes following 3 x ABVD and 30 Gy IFRT (5-year event-free survival rates 95.3\% and $93.5 \%$, respectively), thus may potentially be spared the additional toxicity of escalated BEACOPP, whilst those with Deauville score 5 have a much higher relapse risk [11].

\section{Personalised Approaches to Treatment}

PET-adapted trials provide important information regarding the risk of disease relapse with CMT and chemotherapy approaches, but consideration of the risks of early and late toxicity is equally as important in determining the optimum treatment approach. Radiation field, dose, sex, co-morbidities and age are all important considerations. A large study of HL survivors has shown secondary malignancy rates of up to $33 \%$ at 30 years, noting that many will have received more extended-field radiotherapy than is current practice. The standard incidence ratio of cancer was highest in those diagnosed aged 15-35 years, particularly with respect to breast cancer risk in females receiving mediastinal radiotherapy [24,25]. Similarly, in a study of teenage and young adult cancer survivors, the standardised mortality ratio for cardiac-related death was 3.8 for those diagnosed with $\mathrm{HL}$, with the greatest relative and absolute increase in risk for those aged 15-19 years at diagnosis [26].

Given that the large RT fields and higher radiation doses $(>40$ Gy) of the past were largely implicated in the late side effects of the survivors, newer RT techniques, improvements in advanced RT delivery and substantial reductions in the RT field size and dose are now used. The International Lymphoma Radiation Oncology Group have led the current international standard of care, which is Involved Site Radiation Therapy (ISRT) [27]. ISRT represents a large normal tissue volume reduction from the previous wide RT treatment fields of the past $[28,29]$, which is anticipated to reduce, but not eliminate, late toxicity. 
These trials confirm that CMT provides the best disease control and help clinicians to quantify the risk of omitting RT when informing patients. Nevertheless, a PET-based approach using chemotherapy alone for those achieving CMR remains an entirely reasonable option for selected patients based on their individual characteristics or treatment goals. The decision about whether to omit RT after chemotherapy provides an insight into the challenges of modern effective cancer medicine, where life beyond cure is now a key consideration for most patients. Randomised trial results suggest that some patients can be safely treated with chemotherapy alone and these results inform the patient-specific discussion about the increased risk of relapse omitting RT. Examples where omitting RT may be the preferred option include a young woman with axillary or mediastinal disease where receiving RT to axillary and mediastinal lymph nodes may necessitate irradiating breast tissue and substantially increase the risk of breast cancer, or where cardiac structures may receive clinically significant RT doses in addition to the potential cardiac toxicity of anthracycline-based therapies. In these patients, the individual risk of relapse and the risk of RT-associated late effects including secondary cancer should be carefully considered. In many older or co-morbid patients, minimising acute chemotherapy toxicity may be more of a concern, and RT can be useful in reducing the risk of relapse and need for salvage therapies.

Of paramount importance indecision making in early stage HL is the patient's informed choice as to whether a 3.6 to upto $12.1 \%$ reduction in the rate of relapse with the addition of RT is worth the potential risk of additional late toxicity, which often occurs decades after delivering the RT. Some patients will elect to receive RT because they do not want to live with the increased short term risk of relapse. Others will elect to minimise the long term risks of radiotherapy and accept an increased risk of local relapse, where late radiation toxicity, such as effects on the heart and the risk of a second cancer or breast cancer in younger women, are of greater concern [30]. Based on current evidence, CMT is preferred for patients at highest risk of relapse, or where the anticipated risk of late radiation toxicity is low or negligible. However, chemotherapy alone confers excellent overall survival rates and is a valid option for selected patients, particularly where the late toxicity risks of RT may be higher.

Can modelling help with this complex clinical risk decision making in evaluating the risks and benefits with these different treatment options and the important question of whether to consolidate with RT? Early attempts to do this have started [31], although this type of modelling is certainly not ready for routine clinical application at present. Whatever model is used can only be a reflection of the input data, for example, much of the long-term outcome data is derived from older studies using more extensive radiation fields [29]. Importantly, when complex clinical outcome scenarios are modelled, often using study-level rather than individual patient data, a multitude of underlying assumptions must be made.

In summary, considerable progress has been made in moving towards personalising treatment approaches in early-stage $\mathrm{HL}$ and in moving away from "one size fits all" with the same treatment approach for each clinical risk group. However, we currently have much more work to do before we are able implement this personalised approach for all patients. Currently discussing and agreeing the most appropriate treatment approach can be amongst the most difficult parts of decision making for the both haemato-oncologist, radiation oncologist and the patient. We are now making progress in moving from long established pre-treatment risk stratification dictating management approaches to integrate response-adapted approaches with PET alongside patient-specific characteristics of age, sex, site of disease and patient preferences. Only such a balanced, integrated and informed approach with long term detailed follow-up will lead to the personalised care required to further improve outcomes in early-stage HL.

\section{References}

1. Kaplan HS (1996) Role of intensive radiotherapy in the management of Hodgkin's disease. Cancer 19: 356-367.

2. Peters MV, Middlemiss KC (1958) A study of Hodgkin's disease treated by irradiation. Am J Roentgenol Radium. Ther Nucl Med 79: 114-121. [crossref]

3. Aleman BM, van den Belt-Dusebout AW, Klokman WJ, Van't Veer MB, Bartelink $\mathrm{H}$, et al. (2003) Long-term cause-specific mortality of patients treated for Hodgkin's disease. J Clin Oncol 21: 3431-3439. [crossref]

4. Ng AK, Bernardo MP, Weller E, Backstrand KH, Silver B, et al. (2002) Long-term survival and competing causes of death in patients with early-stage Hodgkin's disease treated at age 50 or younger. J Clin Oncol 20: 2101-2108. [crossref]

5. Engert A, Franklin J, Eich HT, Brillant C, Sehlen S, et al. (2007) Two Cycles of Doxorubicin, Bleomycin, Vinblastine, and Dacarbazine Plus Extended-Field Radiotherapy Is Superior to Radiotherapy Alone in Early Favorable Hodgkin's Lymphoma: Final Results of the GHSG HD7 Trial. Journal of Clinical Oncology 25: 3495-3502. [crossref]

6. Fermé C, Eghbali H, Meerwaldt JH, Rieux C, Bosq J, et al. (2007) Chemotherapy plus involved-field radiation in early-stage Hodgkin's disease. N Engl J Med 357: 19161927. [crossref]

7. Noordijk EM, Carde P, Dupouy N, Hagenbeek A, Krol AD, et al. (2006) Combinedmodality therapy for clinical stage I or II Hodgkin's lymphoma: long-term results of the European Organisation for Research and Treatment of Cancer H7 randomized controlled trials. J Clin Oncol 24: 3128-3135. [crossref]

8. Engert A, et al. (2010) Reduced treatment intensity in patients with early-stage Hodgkin's lymphoma. N Engl J Med 363: 640-652.

9. Eich HT, et al. (2010) Intensified chemotherapy and dose-reduced involved-field radiotherapy in patients with early unfavorable Hodgkin's lymphoma: final analysis of the German Hodgkin Study Group HD11 trial. J Clin Oncol 28: 4199-4206.

10. Klimm B, Goergen H, Fuchs M, von Tresckow B, Böll B, et al. (2013) Impact of risk factors on outcomes in early-stage Hodgkin's lymphoma: an analysis of international staging definitions. Ann Oncol 24: 3070-3076. [crossref]

11. Barrington SF, Phillips EH, Counsell N, Hancock B, Pettengell R, et al. (2019) Positron Emission Tomography Score Has Greater Prognostic Significance Than Pretreatment Risk Stratification in Early-Stage Hodgkin Lymphoma in the UK RAPID Study. J Clin Oncol 37: 1732-1741. [crossref]

12. Illidge TM, Phillips EH, Counsell N, Pettengell R, Johnson PWM, et al. (2020) Maximum tumor diameter is associated with event-free survival in PET-negative patients with stage I/IIA Hodgkin lymphoma. Blood Advances 4: 203-206. [crossref]

13. Kumar A, Burger IA, Zhang Z, Drill EN, Migliacci JC, et al. (2016) Definition of bulky disease in early stage Hodgkin lymphoma in computed tomography era: prognostic significance of measurements in the coronal and transverse planes. Haematologica 101: 1237:1243. [crossref]

14. Cottereau A-S, Versari A, Loft A, Casasnovas O, Bellei M, et al. (2018) Prognostic value of baseline metabolic tumor volume in early-stage Hodgkin lymphoma in the standard arm of the H10 trial. Blood 131: 1456-1463. [crossref]

15. Mottok A, Steidl C (2018) Biology of classical Hodgkin lymphoma: implications for prognosis and novel therapies. Blood 131: 1654-1665. [crossref] 
16. Hsi ED, Li H, Nixon AB, Schöder H, Bartlett NL, et al. (2019) Serum levels of TARC, MDC, IL-10, and soluble CD163 in Hodgkin lymphoma: a SWOG S0816 correlative study. Blood 133: 1762-1765. [crossref]

17. Spina V, Bruscaggin A, Cuccaro A, Martini M, Di Trani M, et al. (2018) Circulating tumor DNA reveals genetics, clonal evolution, and residual disease in classical Hodgkin lymphoma. Blood 131: 2413-2425. [crossref]

18. Gallamini A, Hutchings M, Rigacci L, Specht L, Merli F, et al. (2007) Early Interim 2-[18F] Fluoro-2-Deoxy-D-Glucose Positron Emission Tomography Is Prognostically Superior to International Prognostic Score in Advanced-Stage Hodgkin's Lymphoma: A Report From a Joint Italian-Danish Study. Journal of Clinical Oncology 25: 3746-3752. [crossref]

19. André MPE, Girinsky T, Federico M, Reman O, Fortpied C, et al. (2017) Early Positron Emission Tomography Response-Adapted Treatment in Stage I and II Hodgkin Lymphoma: Final Results of the Randomized EORTC/LYSA/FIL H10 Trial. J Clin Oncol 35: 1786-1794. [crossref]

20. Straus DJ, Jung SH, Pitcher B, Kostakoglu L, Grecula JC, et al. (2018) CALGB 50604: risk-adapted treatment of nonbulky early-stage Hodgkin lymphoma based on interim PET. Blood 132: 1013-1021. [crossref]

21. Fuchs M, Goergen H, Kobe C, Kuhnert G, Lohri A, et al. (2019) Positron Emission Tomography-Guided Treatment in Early-Stage Favorable Hodgkin Lymphoma: Final Results of the International, Randomized Phase III HD16 Trial by the German Hodgkin Study Group. Journal of Clinical Oncology 37: 2835-2845. [crossref]

22. Radford J, Illidge T, Counsell N, Hancock B, Pettengell R, et al. (2015) Results of a Trial of PET-Directed Therapy for Early-Stage Hodgkin's Lymphoma. New England Journal of Medicine 372: 1598-1607.

23. Raemaekers JMM, André MP, Federico M, Girinsky T, Oumedaly R, et al. (2014) Omitting Radiotherapy in Early Positron Emission Tomography-Negative Stage I/II Hodgkin Lymphoma Is Associated With an Increased Risk of Early Relapse: Clinical Results of the Preplanned Interim Analysis of the Randomized EORTC/LYSA/FIL H10 Trial. Journal of Clinical Oncology 32: 1188-1194. [crossref]
24. Schaapveld M, et al. (2015) Second Cancer Risk Up to 40 Years after Treatment for Hodgkin's Lymphoma. New England Journal of Medicine 373: 2499-2511.

25. Sud A, Thomsen H, Sundquist K, Houlston RS, Hemminki K (2017) Risk of Second Cancer in Hodgkin Lymphoma Survivors and Influence of Family History. Journal of Clinical Oncology 35: 1584-1590.

26. Henson KE, Reulen RC, Winter DL, Bright CJ, Fidler MM, et al. (2016) Cardiac Mortality Among 200000 Five-Year Survivors of Cancer Diagnosed at 15 to 39 Years of Age: The Teenage and Young Adult Cancer Survivor Study. Circulation 134: 15191531. [crossref]

27. Illidge T, Specht L, Yahalom J, Aleman B, Berthelsen AK, et al. (2014) Modern Radiation Therapy for Nodal Non-Hodgkin Lymphoma\&\#x2014; Target Definition and Dose Guidelines From the International Lymphoma Radiation Oncology Group. International Journal of Radiation Oncology, Biology, Physics 89: 49-58. [crossref]

28. Engert A, et al. (2003) Involved-field radiotherapy is equally effective and less toxic compared with extended-field radiotherapy after four cycles of chemotherapy in patients with early-stage unfavorable Hodgkin's lymphoma: results of the HD8 trial of the German Hodgkin's Lymphoma Study Group. J Clin Oncol 21: 3601-3608.

29. Meyer RM, et al. (2011) ABVD Alone versus Radiation-Based Therapy in LimitedStage Hodgkin's Lymphoma. New England Journal of Medicine 366: 399-408.

30. Bröckelmann PJ, McMullen S, Wilson JB, Mueller K, Goring S, et al. (2019) Patient and physician preferences for first-line treatment of classical Hodgkin lymphoma in Germany, France and the United Kingdom. British Journal of Haematology 184: 202214. [crossref]

31. Parsons SK, Kelly MJ, Cohen JT, Castellino SM, Henderson TO, et al. (2018) Early-stage Hodgkin lymphoma in the modern era: simulation modelling to delineate long-term patient outcomes. British Journal of Haematology 182: 212221. [crossref]

\section{Citation:}

Tim Illidge and Elizabeth H Phillips (2020) Personalised Approaches in Treating Early-Stage Hodgkin Lymphoma. Internal Med Res Open J Volume 5(3): 1-4. 